\title{
Implementación de una estación retransmisora de radio en FM para una red satelital regional en el Nororiente Peruano
}

\section{Implementation of a retransmission station of FM radio for a regional satellite network in the Northeastern Peruvian}

\author{
Julio Ernesto Quispe Rojas ${ }^{1}$, Herless H. Alberca Vásquez² ${ }^{2}$ Denis Amari Maldonado ${ }^{3}$
}

${ }^{1}$ Escuela Profesional de Ingeniería Electrónica, Facultad de Ciencias Físicas y Matemáticas

Universidad Nacional Pedro Ruiz Gallo, Av. Juan XXIII No. 391 - Lambayeque - Perú.

${ }^{2} y^{3}$ Radio Marañon, Av. Orellana No. 343 Jaen - Peru.

\section{RESUMEN}

Los sistemas de radiodifusión comercial, a mediados del siglo $\mathrm{XX}$, se realizaban utilizando la modulación por amplitud - AM y las radioemisoras generaban la señal en forma local para transmitirla directamente a su área de influencia.

En la segunda mitad del siglo XX, la transmisión en Frecuencia Modulada - FM, se extendió rápidamente por la calidad del sonido, que es una señal con dos canales, izquierdo y derecho, y en la ultima década del siglo $X X$, en nuestro país se utilizaban enlaces satelitales y retransmisores en FM, formando cadenas nacionales de radiodifusión, y lograron la mayor sintonía de los radioescuchas de provincias.

Los costos para subir la señal al satélite, se podía asumir si se requería mas de doce estaciones repetidoras, sin embargo con el desarrollo tecnológico, en la actualidad esta al alcance de los empresarios e instituciones de provincias, para formar cadenas regionales, con la señal generada en la ciudad principal de la zona, con información de interés local y con una programación orientada a satisfacer los gustos de la región.

El proyecto satisface la necesidad de ampliar la cobertura y mejorar la calidad de señal, y obedeciendo al reto de que la señal transmitida llegue a los distritos de la provincia y a las provincias vecinas, y la calidad del sonido sea estereofónico, utilizando para ello estaciones de retransmisión en FM, formando una Red Satelital Regional en el Nororiente del Perú, en la zona norte del Departamento de Cajamarca y Amazonas, en la frontera con el vecino país del Ecuador.

El proyecto de está enfocado a aprovechar el sistema satelital que cuenta la organización, formada por una estación de transmisión hacia el satélite o Up Link, y tan solo una estación de recepción y de retransmisión en amplitud modulada - AM, ubicadas en la provincia de Jaén, Departamento de Cajamarca.

El artículo que presentamos permite compartir la experiencia que se ha tenido en el diseño e implementación de la estación retransmisora en FM para la Red Satelital Regional, implementando la estación de recepción vía satélite y transmisión en FM en el Distrito de Chirinos, en la Provincia de San Ignacio, en el Departamento de Cajamarca.

Descriptores: Radio en FM, Enlace vía satélite para radiodifusión, Estación de retransmisión.

\section{ABSTRACT}

Commercial broadcasting systems in the mid-twentieth century, were performed using amplitude modulation $\mathrm{AM}$ and the radio signal generated locally to transmit directly to their area of influence.

In the second half of the twentieth century, the transmission frequency modulation - FM, it quickly spread about the quality of sound, which is a signal with two channels, left and right, and in the last decade of the twentieth century in our country using satellite links and FM broadcasters, making national radio, and achieved the highest-rated radio listeners in the provinces. 
The costs for the satellite uplink, you could take if they needed more than a dozen base stations, however with technological development, now is accessible to entrepreneurs and provincial institutions to form regional channels, with signal generated in the main town in the area, with information of local interest and with a program aimed at satisfying the tastes of the region.

The project addresses the need to expand coverage and improve signal quality, and obeying the challenge of the transmitted signal reaches the districts of the province and neighboring provinces, and stereo sound quality, using stations FM broadcast, forming a Regional Satellite Network in the Northeast of Peru, in the northern department of Cajamarca and Amazonas, on the border with the neighboring country of Ecuador.

The project is aimed to harness satellite system that has the organization, formed by a transmission station to the satellite or Link Up, and only one receiving station and amplitude modulated broadcasting - AM, located in the province of Jaén, Department of Cajamarca.

The present article to share the experience has had on the design and implementation of the FM relay station for Regional Satellite Network, implementing the satellite receiving station and FM broadcasting in the District of Chirinos, in the Province San Ignacio, in the Department of Cajamarca.

Keywords: FM radio; broadcasting via satellite link, retransmission station.

\section{INTRODUCCIÓN}

La radiodifusión comercial y educativa, en las provincias del Perú, son atendidas en la actualidad por cadenas nacionales de radiodifusión, que tienen filiales propias o filiales asociadas, que cuentan con una estación terrena vía satélite para la recepción de la señal, una planta transmisora en frecuencia modulada - FM y en algunos casos estudios para programas propios y publicidad radial, y por radioemisoras locales, que tiene una planta transmisora en FM y estudios para producir los programas y la publicidad radial que emitirán.

Los sistemas de radiodifusión a mediados del siglo $X X$, se realizaban utilizando la modulación por amplitud - AM, la señal de audio era solo monoaural, y las radioemisoras generaban la señal en forma local para transmitirla directamente a su área de influencia.

En la segunda mitad del siglo $\mathrm{XX}$, la transmisión en Frecuencia Modulada - FM, se extendió rápidamente por la calidad del sonido, que es una señal estereofónica, vale decir, con dos canales de audio, izquierdo y derecho, que es la forma natural como captan nuestros dos oídos los sonidos de las diferentes fuentes: música, conferencias, conversaciones en general.

En la década de los años ochenta se inicia en el Perú el uso de los enlaces vía satélite para la recepción y retransmisión de las señales de televisión, y en la década de los años noventa, se inicia el uso de estos enlaces para la recepción y retransmisión de radioemisoras, principalmente en FM, formándose las cadenas nacionales de Radio, cuyas principales cadenas en el actualidad son: Grupo RPP, Corporación Radial del Perú, Coordinadora Nacional de Radio CNR, etc.

La evolución de la difusión de música, noticias, y programas educativos en las provincias del Nororiente, que no difiere mucho del resto de las provincias del Perú, se inicia con la instalación de parlantes en postes de las plazas y mercados, y mediante una consola, un excitador $y$ un amplificador de potencia de audio, se contribuye a la diversión e información.

Luego, se progresa a la instalación de una planta transmisora en FM, que debido al avance de la tecnología electrónica, tiene costos accesibles para los pequeños empresarios de radiodifusión de provincias, en algunos casos, estos empresarios hacen alianza con las cadenas nacionales para usar su señal compartiendo con su propia programación y publicidad.

Algunas cadenas nacionales instalan sus propias filiales, sobre todo en las localidades de mayor movimiento comercial, que les permite exhibir, en las reuniones de preventa de publicidad, la cobertura de la cadena radial, y de esta manera la señal nacional de emisoras de Lima, llegan a las provincias. 
Existen pocas experiencias de señales de provincias se emitieron a nivel nacional mediante una cadena nacional con estudios principales en provincias, dos de estas experiencias fueron: Radio Hit, cuya señal subía al satélite en Ica, y tenía estaciones retransmisores en las doce principales ciudades del Perú, y el otro fue Radio Universal de Monsefu, en Lambayeque, que tuvo pocas estaciones retransmisoras.

Los costos para subir la señal al satélite, se podía asumir si se requería para doce o más estaciones repetidoras, sin embargo con el desarrollo tecnológico, en la actualidad esta al alcance de los empresarios e instituciones de provincias, para formar cadenas regionales, con la señal generada en la ciudad principal de la zona, con información de interés local y con una programación orientada a satisfacer los gustos de la región, y estaciones retransmisoras en los distritos y provincias vecinas.

¿Cómo se puede implementar una estación retransmisora de FM para una Red Satelital Regional? Es la pregunta que motiva la presente investigación, que se justifica por la necesidad de integrar a los pueblos cercanos, que siempre estuvieron identificados y comunicados entre ellos, a pesar de las limitaciones del lento progreso de nuestro país.

Las comunicaciones escritas y verbales que existían entre los pueblos, fueron mejorados por la radio en onda media con amplitud modulada, que tiene mayor cobertura que las radios en FM, por que usan ondas de tierra, en lugar de ondas por líneas de vista que utilizan las emisoras en FM; y de pronto la música, las noticias de la capital son compartidas en los pueblos en lugar de las noticias y gustos musicales propios de la zona, esto exige la coexistencia con las cadenas radiales nacionales y redes regional de radioemisoras en $\mathrm{FM}$, así como radioemisoras locales.

El estudio tiene por objetivo conocer las etapas y elementos de la Implementación de una estación de retransmisión de radioemisora en FM para una red satelital regional en el Nororiente Peruano, que comprende el diseño del proyecto y las instalación de los equipos, pruebas y puesta en servicios, que incluye presupuesto y cronograma de ejecución de acuerdo a lo ocurrido en la realidad.

El presente articulo esta basado en la experiencia de los autores en la implementación de diversas estaciones de retransmisión en FM, principalmente la implementación de la estación de retransmisión de Chirinos, de la provincia de San Ignacio,
Departamento de Cajamarca, en la frontera Norte del Perú con el Ecuador; parte de la Red Satelital Regional en el Nororiente Peruano, cuyo crecimiento debe incluir en el futuro próximo retransmisoras en Santa María de Nieva y en Sallique, en los Departamentos de Amazonas y Cajamarca respectivamente.

El proyecto de está enfocado a aprovechar el sistema satelital con el que cuenta la organización y que está conformada por una estación de transmisión hacia el satélite o Up Link, y una estación de recepción y de retransmisión en Amplitud modulada, ubicadas en la provincia de Jaén, Departamento de Cajamarca, y satisface la necesidad de ampliar la cobertura y mejorar la calidad de señal, y obedeciendo al reto de que la señal transmitida llegue a otras provincias y con la calidad de sonido estereofónico.

\section{PLANTEAMIENTO EXPERIMENTAL}

El proceso experimental del estudio realizado comprende el Diseño del Proyecto y la Ejecución del Proyecto, para la Implementación de una Estación Retransmisora de Radio en FM, que forma parte de una Red Satelital Regional de Radiodifusión de una organización en el Nororiente Peruano; el proyecto incluye dos partes: el sistema de recepción vía satélite y el sistema de transmisión de radio en FM.

Para el diseño se debe determinar en primer lugar el tipo de enlace satelital a utilizar y luego seleccionar la ubicación de la Estación y basado en ello seleccionar los elementos que se utilizaran para el sistema de retransmisión en FM, sus presupuesto y cronograma de ejecución tentativo, tramites de autorizaciones, cotizaciones, financiamiento.

Para la instalación de los equipos, se debe verificar la disponibilidad de los equipos y prever la oportuna culminación de las etapas previas de la Infraestructura, y conocimientos y habilidades para la instalación y pruebas de funcionamiento, ajustes y puesta en operación definitiva, que se debe realizar después de subsanar las observaciones y completar de los documentos definitivos del proyecto, que incluye planos modificados de acuerdo a la realidad y presupuesto, catálogos y cronogramas reales de lo ejecutado.

El enlace típico para una red suele ser satelital, teniendo en cuenta que los sistemas vía satélite son capaces de proveer servicios de comunicaciones virtualmente a cualquier parte del mundo sin discriminación en precios o geografía. Ninguna otra 
tecnología -incluyendo fibra óptica- puede conseguir este objetivo y ninguno puede lograr la promesa de universalidad geográfica [1].

Para seleccionar el satélite se tiene en cuenta el uso en la región y facilidad para los contratos, INTELSAT es el más grande consorcio global mundial de comunicaciones por satélite conformado por más de 140 países miembros. En la actualidad controla 19 satélites, uno de los utilizados es el satélite Pas $1 \mathrm{R}$ [2].

La tecnología seleccionada es la de un solo canal por portadora, SCPC (Single Channel Per Carrier), esta tecnología que permite la transmisión de datos, voz, video, y consiste en transmitir una señal digital en una frecuencia fija, llamada portadora, el equipamiento de la unidad terrestre SCPC consta de una unidad de antena y un sistema de transmisión que incluye el amplificador de potencia (SSPA), el amplificador de bajo ruido (LNB) y el Up/Down Converter, y el sistema de recepción integra un módem satelital con tarjeta moduladora y tarjeta demoduladora, dispositivo de control y circuitos de procesamiento de señal e interfaz para interconexión con el usuario [3].

La Radio en Frecuencia Modulada - FM, la señal de radio o portadora está modulada en frecuencia, que permite difundir información de audio para ser recepcionada en forma libre y directa por el público en general, la propagación es por Onda Directa y la Banda de Operación: $88 \mathrm{MHz}$ a $108 \mathrm{MHz}$ [4].

\section{RESULTADOS Y DISCUSIÓN}

\section{DISEÑO DE LA ESTACION RETRANSMISORA DE RADIO EN FM}

\section{Selección de la ubicación de la Estación}

La localización de la estación terrena de recepción y de transmisión en FM, está ubicada en la parte más alta del cerro La cordillera, en el distrito de Chirinos, el lugar permite la visibilidad a varios distritos de la provincia de San Ignacio por encontrarse en la parte más alta, tiene un fácil acceso por carretera y suministro de energía eléctrica comercial.

Las coordenadas del sitio de la implementación del proyecto, se obtuvo con la ayuda de un GPS y son las siguientes: Latitud sur: $5^{\circ} 17^{\prime} 21.4$ ', Longitud oeste: $78^{\circ} 54^{\prime} 11.6^{\prime \prime}$, Altura: 2,024 msnm.

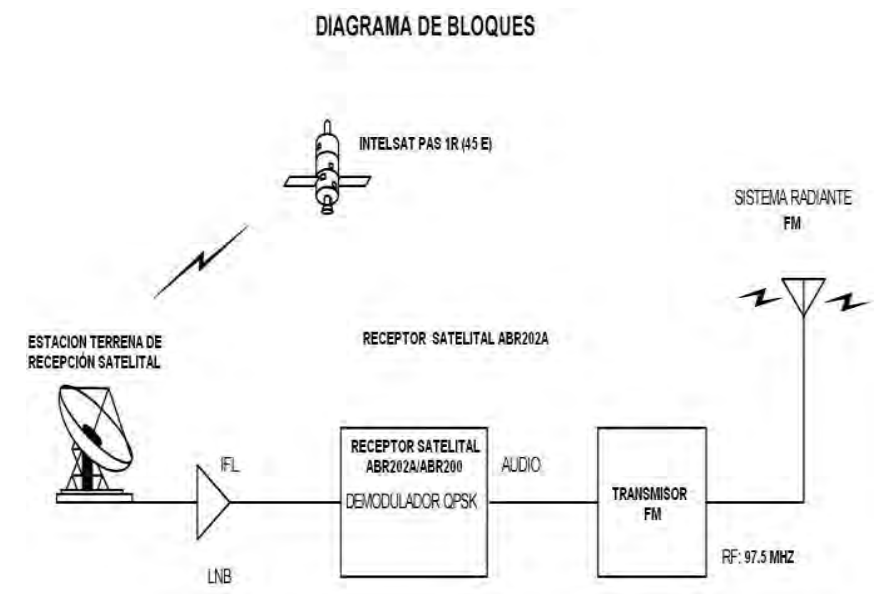

Figura 1: Diagrama en bloques del sistema de recepción satelital y retransmisión de radio en FM

\section{Sistema de recepción satelital}

El sistema está constituido por los siguientes elementos: Antena para recepción satelital, Amplificador de bajo ruido, Receptor de audio satelital.

La selección de la antena parabólica, se realiza basándose en la recomendación de INTELSAT para la Banda $C$ de satélite, y puede variar entre: 2.4 metros, 3.8 metros, y 4.5 metros.

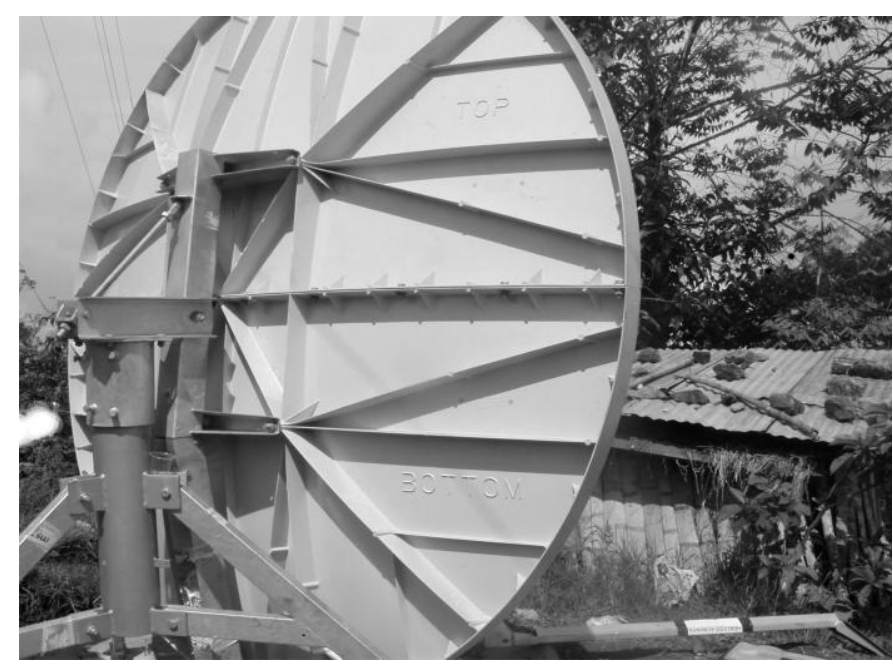

Figura 2: Antena Parabólica Vista posterior

Se seleccionó una antena de 2.4 metros, Marca Channel Master/Andrew de procedencia U.S.A. Ganancia TX: $42.0 \mathrm{dBi}$ y RX: $38.0 \mathrm{dBi}$, polarización lineal, montaje $A Z / E L$ azimut $360^{\circ}$ y elevación de $10^{\circ}$ a $90^{\circ}$, Frecuencias: TX: 5.850 a $6.725 \mathrm{GHz}, \mathrm{RX}$ : 3.400 a $4.200 \mathrm{GHz}$., OFFSET de $22.6^{\circ}$. 


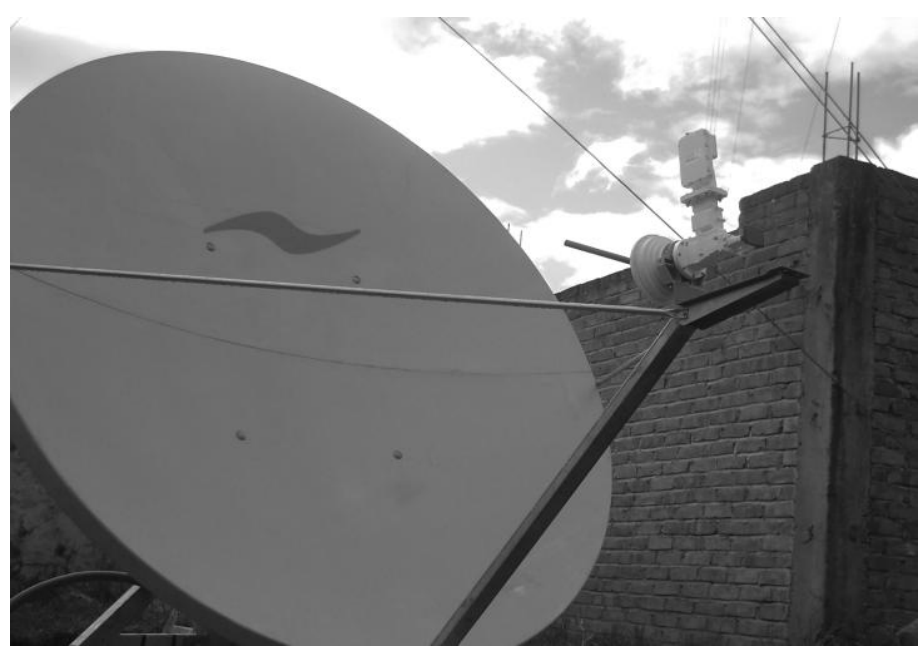

Figura 3: Antena Parabólica Vista frontal

El Amplificador de Bajo Ruido - LNB, debe tener las características de compatibilidad con el alimentador de la antena, y capacidad para recibir señales de audio digital comprimido y moduladas en fase, para ello el LNB posea bucle de enganche de fase PLL.

Se eligió un PLL LNB, Marca JRC, Modelo NJS8476E de fabricación Japonesa, para trabajar en Banda C, con oscilador local de $5.15 \mathrm{GHz}$, una temperatura de ruido de $30^{\circ} \mathrm{K}$, margen de salida de 950 a $1750 \mathrm{MHz}$.

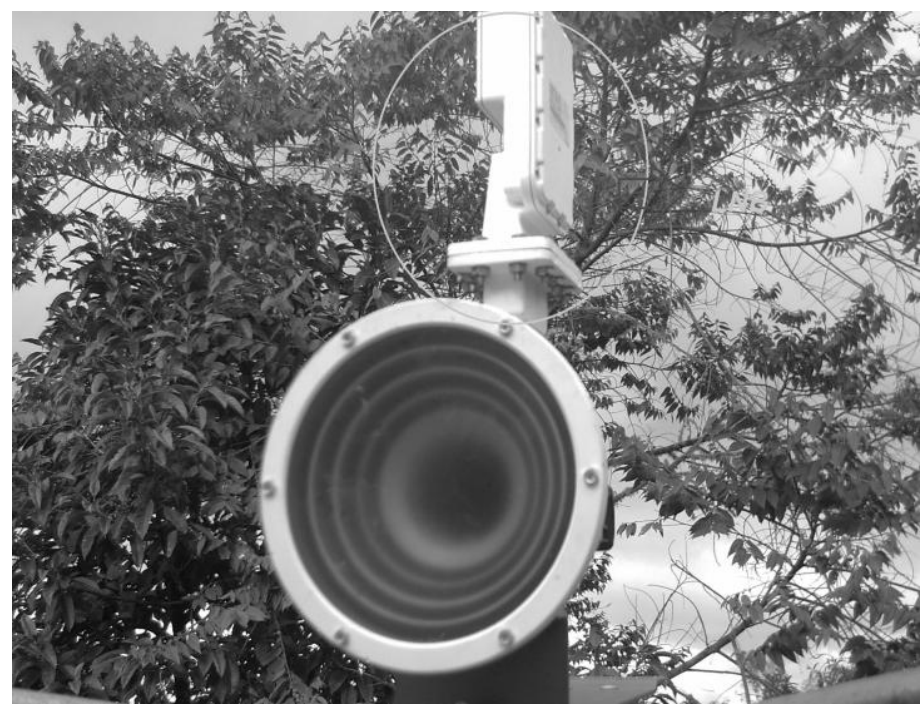

Figura 4: Alimentador ASC S y LNB JRC NJS8476E

El Alimentador seleccionado es de la marca ASC Signal, modelo 611618421, especial para parabólicas o de reflectores con OFFSET, para condiciones climáticas de zona lluviosa y húmeda, y con frecuencia de operación dentro del rango de la antena parabólica y el LNB.

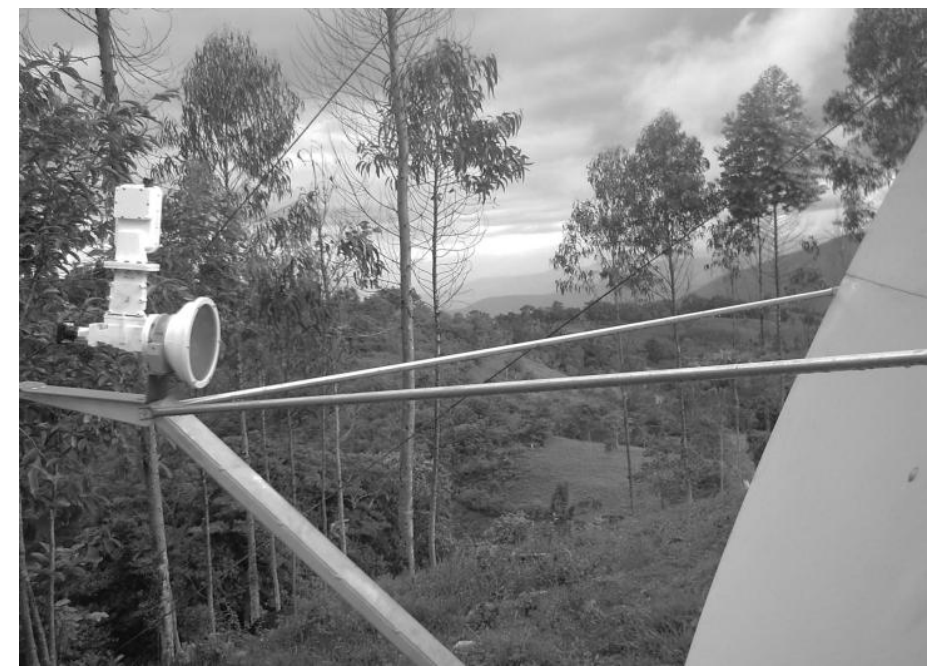

Figura 5: Alimentador y LNB instalado

El Receptor Satelital o modem satelital, marca Tiernan Radyne, modelo ABR202A, es un receptor de audio digital para la recepción de audio de alta fidelidad vía satélite, con interfase grafica, para configurar el enlace, visualizando los principales parámetros del enlace, entre ellos la potencia que se está recibiendo del satélite

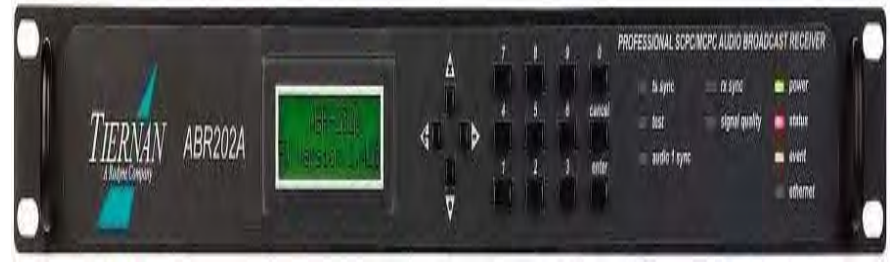

Figura 6: Modem satelital ABR202A Vista frontal

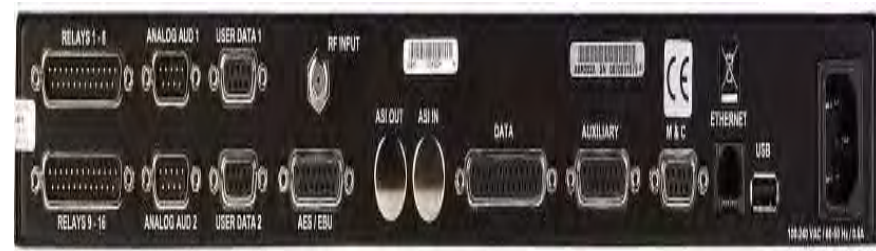

Figura 7: Modem satelital ABR202A Vista posterior

\section{Sistema de transmisión de Radio en FM}

Para seleccionar un transmisor, se debe tener en cuenta el costo, tecnología, garantía, repuestos y soporte técnico, que se ajusten a las normas establecidas por la UIT- R, transmisor de 500 vatios, elegimos el transmisor de la marca DB ELETTRONICA, compuesto por un Excitador FM Stereo de 30W en estado sólido modelo DPM-30-S y un Amplificador de potencia de $500 \mathrm{w}$ de salida modelo KFS 500. 


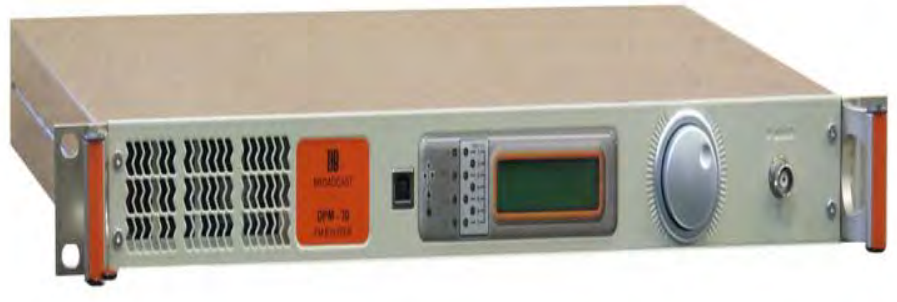

Figura 8: Excitador FM estéreo de 30W-DPM 30 S

El Excitador FM Stereo, marca DB ELETTRONICA, modelo DPM 30S, es un excitador diseñado para funcionar en una gama de frecuencias que va desde $87-108 \mathrm{MHz}$ para las aplicaciones de radio de la difusión de FM. Es de potencia de salida RF ajustable a partir de la 0 a $30-60$ - 120 vatios por control externo, ideal como transmisor independiente o el excitador versátil para los amplificadores de Potencia.

El Amplificador de Potencia marca DB ELETTRONICA, modelo KFS 500, de 500 watts de salida, de tecnología Cold FET de alta eficiencia, característico de los transmisores de FM de la serie PF y PM de bajo consumo energía y reducida disipación de calor, una confiabilidad extremadamente alta con dos niveles de redundancia, basados en módulos de $0.5 \mathrm{KW}$ independientes e intercambiables entre si, aislados uno del otro, extraíbles y sustituibles aún con el transmisor en operación; cada módulo está constituido por dos amplificadores de $250 \mathrm{~W}$, independientes y aislados uno del otro, alimentados separadamente.

La potencia de salida de cada módulo es regulada por una lógica "proporcional fold-back" que en caso de problemas con el ROE en antena, se reduce proporcionalmente la potencia sin parar el funcionamiento.

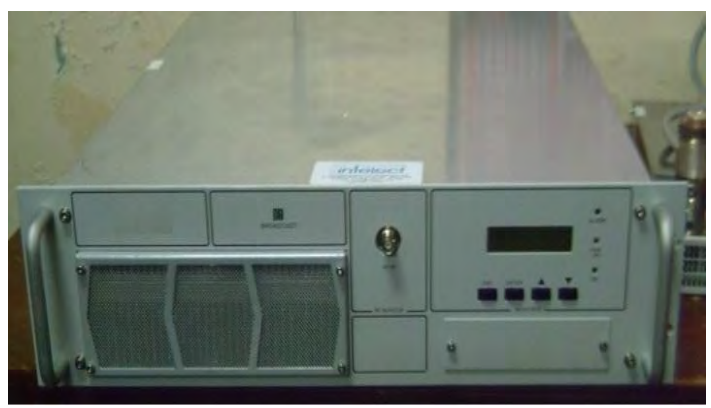

Figura 9: Amplificador de 500W modelo KFS 500

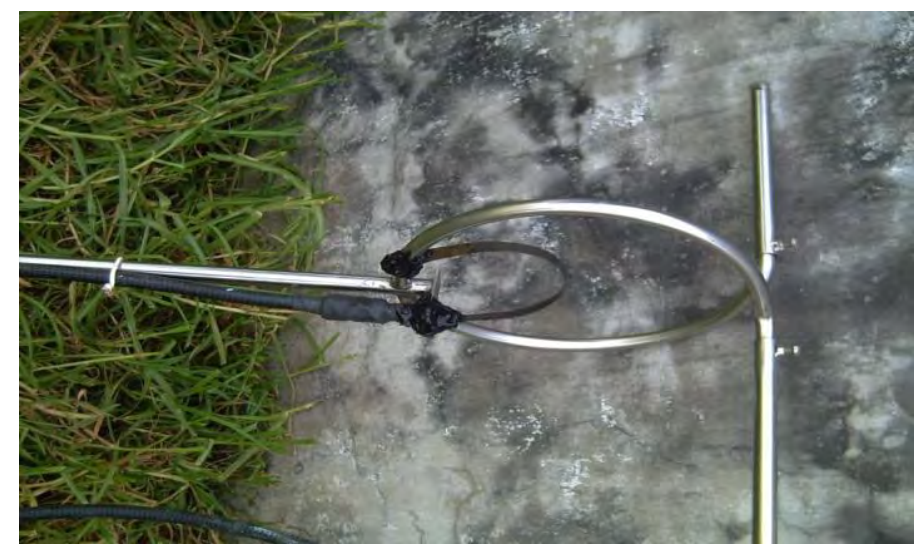

Figura 10: Antenas circular modelo ACPOX41

Las consideraciones para la elección de las antenas y el tamaño de la torra, fueron relieve accidentado, el alto follaje en la zona, no existencia de línea de vista en algunas zonas que se desea cubrir; por ello el sistema de antenas debe ser de buena ganancia, buena radiación y con características eléctricas compatibles con el transmisor; se optó por un sistema radiante de cuatro antenas tipo circular con polarización circular, de uso difundido en la zona por un gran número de radioemisoras.

Elegimos el sistema radiante de 4 antenas FM de polarización circular, marca RVR Electrónica modelo ACPOX41 de fabricación Italiana; compuesto por 04 antenas circulares, modelo ACPO, con dimensiones de $1000 \mathrm{~mm} \times 300 \mathrm{~mm} \times 800 \mathrm{~mm}$. y un distribuidor o divisor de entrada, marca RVR Electrónica brida EIA $7 / 8$ salida $7 / 16$ y 04 latiguillos o jumpers de 5.5 mts. con conectores $7 / 16$.

El cable de conexión del transmisor al distribuidor o divisor con menos perdidas posibles, como cable tipo Heliax LDF5-50 de 7/8" Andrew de diámetro, de impedancia 50 ohms, de 40 metros de longitud; cuya atenuación a la frecuencia operación típica, $f=100$ $\mathrm{Mhz}$, es de aproximadamente $1.19 \mathrm{~dB} / 100 \mathrm{~m}$. Por tanto las pérdidas por cable serán $0.476 \mathrm{~dB}$.

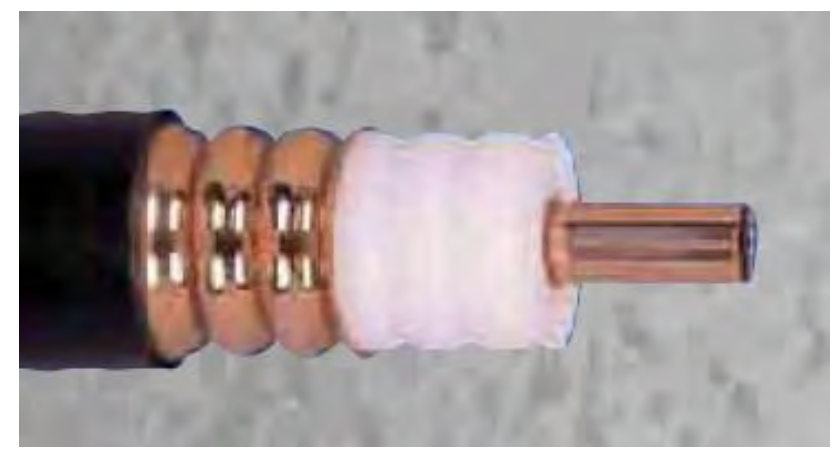

Figura 11: Cable Heliax LDF5-50 de 7/8" Andrew 


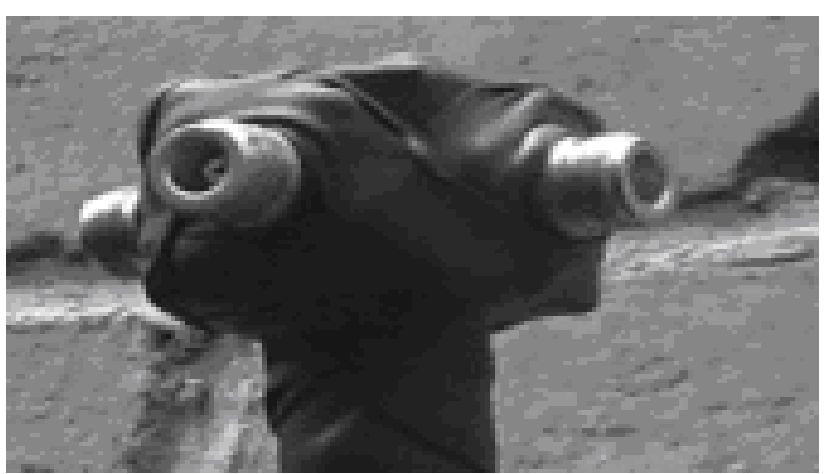

Figura 12: Distribuidor RVR Electrónica

La ubicación de la estación, en la zona alta del cerro la cordillera, no exigía una torre de gran altura, por ello se decidió por una torre de 36 metros, aplicando las recomendaciones de los fabricantes para el peso de las antenas, peso del cable y velocidad máxima del viento, la torre seleccionada es de forma triangular con vientos de acero galvanizado, arriostrada, cada cuerpo es soportado por cuatro triángulos equiláteros de $30 \mathrm{~cm}$. que están en puntos equidistantes de un extremo al otro de cada cuerpo de torre, cada cuerpo de torre de tres metros mas acoplamiento.

\section{Costo del Proyecto}

El costo total de la implementación del proyecto asciende a $\$ 26,310$ incluido IGV, en el monto indicado no se incluye el costo de elaboración del proyecto, trámites de autorizaciones y gastos de transporte y viajes, tampoco obras civiles e instalación de servicios.

Tabla 1: Costo de sistema de recepción vía satélite

\begin{tabular}{|c|c|c|}
\hline ITE & DESCRIPCION & TOTAL \\
\hline 1 & $\begin{array}{l}\text { Kit - Antena Master Channel de } 2.4 \\
\text { metros, marca Andrew }\end{array}$ & 3,600 \\
\hline 2 & Feed Horn - Alimentador & 200 \\
\hline 3 & LNB PLL en banda C & 500 \\
\hline 4 & Receptor de audio ABR202A & 2,000 \\
\hline 5 & Kit de Conectores AF, RF y eléctricos. & 50 \\
\hline 6 & $\begin{array}{l}\text { Cable para audio apantallado Belden } \\
8451\end{array}$ & 30 \\
\hline 7 & Cable RG-8 - 20 metros & 100 \\
\hline 8 & Estabilizador de 8000 watts & 320 \\
\hline 9 & Construcción de pozo de tierra & 280 \\
\hline 10 & $\begin{array}{l}\text { Construcción de una Loza de } \\
12 \text { metros cuadrados para base de } \\
\text { parabólica }\end{array}$ & 250 \\
\hline 11 & $\begin{array}{l}\text { Instalación y configuración de equipos } \\
\text { y estación remota }\end{array}$ & 2000 \\
\hline & $\begin{array}{ll}\text { SISTEMA } & \text { RECEPCION } \\
\text { SATELITE } & \end{array}$ & 9,330 \\
\hline
\end{tabular}

Tabla 2: Costo de implementación de sistema de transmisión de Radio en FM

\begin{tabular}{|c|l|r|}
\hline $\begin{array}{c}\text { IT } \\
\text { E }\end{array}$ & DESCRIPCION & $\begin{array}{l}\text { TOTAL } \\
(\$)\end{array}$ \\
\hline 1 & $\begin{array}{l}\text { Transmisor FM marca DB } \\
\text { ELECTRONICA Modular Compuesto } \\
\text { por: 01 Excitador FM Modelo DPM30; } \\
01 \text { Amplificador KFS 500 }\end{array}$ & 7,000 \\
\hline 2 & $\begin{array}{l}\text { Sistema de Antena marca RVR, } \\
\text { modelo ACPOX41 compuesta de: 4 } \\
\text { antenas ACPOCP, 1 divisor de } \\
\text { potencia PD4NN 1x4 }\end{array}$ & 2,500 \\
\hline 3 & $\begin{array}{l}\text { Torre metálica triangular galvanizada } \\
\text { de 30 cm de lado por 36 metros de } \\
\text { alto incluye accesorios de instalación. }\end{array}$ & 2,500 \\
\hline 4 & \begin{tabular}{l} 
Metros de Cable Heliax de 7/8 \\
\hline 5
\end{tabular} & $\begin{array}{l}\text { Conectores de 7/8 N para cable } \\
\text { Heliax }\end{array}$ \\
\hline 6 & $\begin{array}{l}\text { Inyector y extractor de aire incluido } \\
\text { instalación }\end{array}$ & 320 \\
\hline 7 & $\begin{array}{l}\text { Sistema de pararrayos tetrapuntal } \\
\text { incluye Accesorios, cable de cobre } \\
\text { desnudo 25 mm. Varilla de cobre con } \\
\text { helicoidal más química. }\end{array}$ & 1,000 \\
\hline 8 & $\begin{array}{l}\text { Construcción de bases para vientos y } \\
\text { torre }\end{array}$ & 300 \\
\hline 9 & $\begin{array}{l}\text { Servicio de instalación de torre, } \\
\text { antenas, pararrayos, equipos y otros. }\end{array}$ & 2,400 \\
\hline & $\begin{array}{l}\text { SISTEMA DE TRANSMISION RADIO } \\
\text { FM 16,980 }\end{array}$ \\
\hline
\end{tabular}

\section{INSTALACION DE LA ESTACION RETRANSMISORA DE RADIO EN FM}

Primero se realizó las obras preliminares, para ello se preparó las bases de concreto armado para la torre que soportará a las antenas y las bases para los vientos que dan estabilidad a la torre, y el acondicionamiento de la caseta para los equipos, los pozos de tierra para el sistema de pararrayos y para la protección y referencia de los equipos electrónicos, la loza para base de la antena parabólica.

En segundo lugar se procedió a la instalación del sistema de radiodifusión en $\mathrm{FM}$, montaje de torre, instalación de antenas, distribuidor, cables, excitador y amplificador de potencia, y pruebas de operación. 
Tabla 3: Cronograma real de la ejecución del proyecto

\begin{tabular}{|c|c|c|c|c|c|c|c|c|}
\hline \multirow{3}{*}{ ACTIVIDAD } & \multicolumn{8}{|c|}{ MESES (2010) } \\
\hline & \multicolumn{4}{|c|}{ Mayo } & \multicolumn{4}{|c|}{ Junio } \\
\hline & 1 & 2 & 3 & 4 & 1 & 2 & 3 & 4 \\
\hline $\begin{array}{l}\text { Coordinaciones previos } \\
\text { para la implementación }\end{array}$ & $x$ & & & & & & & \\
\hline $\begin{array}{l}\text { Construcción de la loza, } \\
\text { ductos, bases para } \\
\text { torre. }\end{array}$ & & $x$ & $x$ & & & & & \\
\hline $\begin{array}{l}\text { Acondicionamiento de } \\
\text { caseta y puestas a tierra }\end{array}$ & & & & $x$ & & & & \\
\hline $\begin{array}{l}\text { Instalaciones eléctricas, } \\
\text { e instalación del } \\
\text { inyector y extractor de } \\
\text { aire }\end{array}$ & & & & & x & $\mathrm{x}$ & & \\
\hline $\begin{array}{l}\text { Montaje de torre y } \\
\text { anclado. }\end{array}$ & & & & & & $\bar{x}$ & $x$ & $x$ \\
\hline \multirow[t]{3}{*}{$\begin{array}{l}\text { Instalación de antenas } \\
\text { y Transmisor FM. }\end{array}$} & & & & & & & & $\mathrm{x}$ \\
\hline & \multicolumn{4}{|c|}{ Julio } & \multicolumn{4}{|c|}{ Agosto } \\
\hline & 1 & 2 & 3 & 4 & 1 & 2 & 3 & 4 \\
\hline $\begin{array}{l}\text { Pruebas de desempeño } \\
\text { transmisor y evaluación } \\
\text { de parámetros técnicos. }\end{array}$ & $x$ & $\mathrm{x}$ & & & & & & \\
\hline $\begin{array}{l}\text { Armado de Antena } \\
\text { Parabólica, receptor y } \\
\text { ajustes de enlace }\end{array}$ & & & $\mathrm{x}$ & x & & & & \\
\hline $\begin{array}{l}\text { Pruebas de sistema } \\
\text { satelital y transmisión } \\
\text { FM }\end{array}$ & & & & & $x$ & $x$ & & \\
\hline $\begin{array}{l}\text { Pruebas de emisión de } \\
\text { señal al aire }\end{array}$ & & & & & & & $x$ & \\
\hline $\begin{array}{l}\text { Puesta en Marcha } \\
\text { definitiva }\end{array}$ & & & & & & & & $x$ \\
\hline
\end{tabular}

En tercer lugar se instaló el sistema de recepción vía satélite, antena parabólica, alimentador, LNB, cables, receptor satelital o Modem, y ajuste de apuntamiento, verificación de parámetros y pruebas de operación.

Finalmente se realizaron las pruebas de funcionamiento conjunto, con la señal de la organización transmite desde Jaén, y la puesta en servicio del la estación retransmisora de radio en FM, con la evaluación de la cobertura del distrito de Chirinos y los distritos vecinos.

La implementación se realizó en cuatro meses, de Mayo a Agosto del 2010, tal como se observa en la Tabla 3, cronograma real de ejecución, que es una actualización del cronograma tentativo del proyecto.

\section{CONCLUSIONES}

Los enlaces vía satélite mediante tecnología SCPC, un canal por portadora, permiten el funcionamiento de una Red Satelital Regional de Radio en FM, en los aspectos Técnicos y económicos, cuyos costos se pueden justificar desde dos estaciones retransmisoras, el costo de una estación retransmisora esta en $\$ 26,310$.

La señal se genera en una localidad principal de una región, con información y noticias de la región y con la música que gusta a los pobladores, se transmite mediante Radio en FM para la localidad principal y los lugares cercanos, y la señal se envía al satélite y se recepciona la señal en una amplia zona de cobertura del satélite, dentro de esa zona se puede instalar una estación retransmisora de Radio en FM, y se tiene una señal de calidad con sonido estéreo y con una área de servicio alrededor de la estación repetidora.

\section{AGRADECIMIENTOS}

\section{A la Asociación Civil Radio Marañón Jaén - Cajamarca}

Al Concejo Distrital de Chirinos

Provincia de San Ignacio - Cajamarca

A la Empresa Ingeniería Radio Telecomunicaciones IRT, Lima

\section{REFERENCIAS}

[1] Definición extraída de http://www.intelsat.com

[2] Comunicaciones por satélite, Neri Vela, Rodolfo, Thomson. 2003.

[3]Comunicación por satélite, Rosado Rodríguez, Carlos. Limusa.

[4] Introducción a lo sistemas de comunicación, Stremler, Ferrel. Pearson. 1998.

\section{E-mails:}

jquisper@unprg.edu.pe , heyner2@hotmail.com, damardo@hotmail.com 